\title{
Erratum to: LINE-1 is preferentially hypomethylated within adenomatous polyps in the presence of synchronous colorectal cancer
}

Alice Chu Jiang ${ }^{1}$, Lela Buckingham², William Barbanera ${ }^{2}$, Amoah Yeboah Korang ${ }^{1}$, Faraz Bishehsari ${ }^{1,3}$ and Joshua Melson ${ }^{1,3^{*}}$

\section{Erratum}

After publication of the article [1] it was brought to our attention that one of the authors had their name misspelt. The fifth author should be "Faraz Bishehsari".

\section{Author details}

'Department of Internal Medicine, Rush University Medical Center, 1717 W Congress Parkway, 10 Kellogg, Chicago, IL 60612, USA. ${ }^{2}$ Department of Pathology, Rush University Medical Center, 600 S. Paulina Street, 1014 AAC, Chicago, IL 60612, USA. ${ }^{3}$ Division of Digestive Diseases, Department of Internal Medicine, Rush University Medical Center, 1725 West Harrison, Suite 206, Chicago, IL 60612, USA.

Received: 19 July 2017 Accepted: 20 July 2017

Published online: 27 July 2017

\section{Reference}

1. Jiang A, Buckingham L, Barbanera W, Korang A, Bishesari F, Melson J. LINE-1 is preferentially hypomethylated within adenomatous polyps in the presence of synchronous colorectal cancer. Clinical Epigenetics. 2017;9:1. doi:10.1186/s13148-017-0325-7.

\footnotetext{
* Correspondence: Joshua_Melson@rush.edu

'Department of Internal Medicine, Rush University Medical Center, 1717 W Congress Parkway, 10 Kellogg, Chicago, IL 60612, USA

${ }^{3}$ Division of Digestive Diseases, Department of Internal Medicine, Rush University Medical Center, 1725 West Harrison, Suite 206, Chicago, IL 60612, USA
} 